The International Journal of Flexible Manufacturing Systems, 6 (1994): 99-121

(C) 1994 Kluwer Academic Publishers, Boston. Manufactured in The Netherlands.

\title{
Optimal Door Fitting with Systematic Fixture Adjustment
}

\author{
SHING-KUO WU, S. JACK HU, AND S.M. WU \\ Department of Mechanical Engineering and Applied Mechanics, The University of Michigan, Ann Arbor, \\ MI 48109
}

\begin{abstract}
A systematic approach is presented to obtain the best door gap quality through optimal door fitting in automobile body manufacturing. First, three indexes of gap quality are defined; they are: (1) door gap width deviation relative to design nominal; (2) door gap parallelism; and (3) car-to-car gap consistency. Then the doorfitting problem is formulated into a general constrained optimization problem. The effects of optimal door fitting on the three quality indexes are evaluated through computer simulation. These results provide a lower bound on the design of nominal door gap by considering process capability. Finally, a computer-aided fixture adjustment scheme is developed to orient a door in a body side opening to achieve the optimal fitting. The amount of adjustment, with the desired orientation obtained from optimization, is calculated based on parametrically modeled local surface features of the fixture and the door. The adequacy of door feature modeling is verified through a door-fitting experiment.
\end{abstract}

Key Words: automobile, assembly, part fixturing, optimization.

\section{Introduction}

Among automobile body fit and finish concerns, door-fit problems have been ranked within the top items due to the high warranty cost. An inadequate door fit on the car body will not only cause functional problems such as wind noise, water leakage, and difficulty in closing the door (excessive door closing effort), but also affects the aesthetic value of the vehicle.

The dimensional variations of gap and flush between a body-in-white (BIW) and doors, or between doors, arise from four sources: (1) dimensional variation of the doors; (2) dimensional variations in the BIW openings; (3) variation of the door hanging process; and (4) effects of painting and general assembly. The first two variations are subassembly process variations and need to be improved upstream, and have been addressed by $\mathrm{Hu}, \mathrm{Wu}$ and Wu (1991). This paper will address the improvement of door hanging processes.

Door hanging is one of the major operations on the production floor, either manual or automatic. In manual operation, a door is positioned onto the BIW opening using a hanging fixture, bolted with hinges, and adjusted when necessary upon visual inspection. Adjustments can include bending and twisting the door, or moving the hinges. Problems with this approach are summarized as follows:

1. The spring back after bending the doors may introduce inconsistency and thus may not always be effective. This inconsistency in the current practice of door fitting calls for the development of a systematic hanging fixture adjustment scheme.

2. The capability of correcting the gap deviations through door fitting is not well understood. Consequently, the variational fault sources cannot be effectively located for corrections. 
3. A systematic procedure is not available for door hanging fixture adjustment. The current practice of door hanging fixture adjustment is done by investigating the measurement data followed by a heuristic decision. The investigation and decision-making procedure is time-consuming if not ineffective.

In automatic door hanging, robots are usually used to position the door relative to the door opening, with the end effector working as the hanging fixture. Consistent gap and flush depends on the positioning of the body, the repeatability of the robot, and the variation of subassemblies, i.e., doors and bodies. With in-line measurements for both doors and side openings, flexible assembly systems could be developed to accommodate part-topart variation. Furthermore, optimal door fitting is feasible through the use of controlled fixture adjustment based on a well-defined objective function and constraints.

In this paper, a systematic approach to obtain optimal door fit is presented based on constrained optimization and systematic fixture adjustment. Simulations are used to reveal the relationship between gap quality and part (door and body) variations through optimal door fitting. Results are discussed to facilitate the determination of (1) the necessity of variation reduction on the door and body side opening for a designed gap; and (2) an adequate nominal gap design to accommodate known process capability. The contact surfaces of the locators and the door are then parametrically modeled so that the fixture adjustment can be determined systematically. Experimental results are presented to demonstrate the effectiveness of parametric modeling in door hanging.

\section{Optimal door fitting}

\subsection{Definition of quality indexes}

As measures of the quality of gaps, the following three indexes are used; they are: (1) average gap width deviation (GWD) relative to design intent, i.e., the nominal gap; (2) gap parallelism (GP), i.e., gap width variation from top to bottom of the door; and (3) consistency from car to car. Figure 1 shows a rear door on the body side opening. These three door-fit quality indexes are quantitatively defined as follows:

1. $G W D=$ gap width deviation $=\frac{\sum_{i=1}^{n}\left(x_{i}-\text { nominal gap }\right)}{n}$

2. $G P=$ gap parallelism $=\frac{\sqrt{\sum_{i=1}^{n}\left(x_{i}-{\text { nominal gap })^{2}}^{2}\right.}}{n}$

3. $\sigma_{G W D}=$ variation of gap width from car to car. 


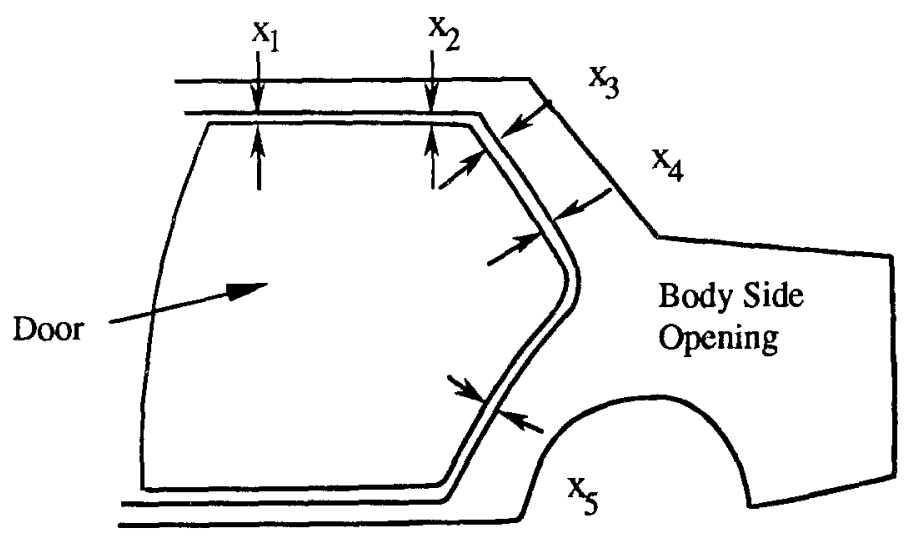

Figure 1. An automobile rear door on the body side opening.

Among the three indexes, GWD and GP are the within-car variation, and $\sigma_{G W D}$ is the carto-car variation. Theoretically, the target values for $G W D, G P$, and $\sigma_{G W D}$ are all zero. However, usually tolerances are specified, and they vary from car to car.

\subsection{Formulation of optimal fitting problem}

The optimal door-fitting problem is formulated as a general constrained optimization problem to orient the door in the three-dimensional space using a homogeneous transformation, such that the objective function is minimized. The calculated transformation variables are then converted to the amount of fixture adjustment.

2.2.1. Homogeneous transformation. A homogeneous transformation matrix $\mathbf{T}$ can be used to represent the relationship between representations of a set of spatial position measurements with respect to either the global reference coordinate or the body frame coordinate (Paul 1981).

$$
\mathbf{T}=\left[\begin{array}{cc}
\mathbf{Q}_{3 \times 3} & \mathbf{D}_{3 \times 1} \\
\mathbf{0}_{1 \times 3} & 1
\end{array}\right]
$$

where $\mathbf{Q}=$ coordinate rotational matrix $; \mathbf{D}=$ coordinate translational matrix $; \mathbf{0}=$ perspective transformation: all zeros for current application; and $1=$ scaling factor: assigned to be one in the current application.

Thus, a spatial vector $\mathbf{U}$ with respect to a coordinate frame $\mathbf{A}$ can be transformed into vector $\mathbf{V}$ with respect to the same coordinate frame $\mathbf{A}$ by premultiplying $\mathbf{U}$ with the homogeneous transformation matrix $\mathbf{T}$, where $\mathbf{T}$ can be shown to be equivalent to a rotation followed by a translation. That is,

$$
\mathbf{V}_{\mathbf{A}}=\mathbf{T} * \mathbf{U}_{\mathbf{A}}=\left[\begin{array}{cc}
\mathbf{Q}_{3 \times 3} & \mathbf{D}_{3 \times 1} \\
\mathbf{0}_{1 \times 3} & 1
\end{array}\right]\left[\begin{array}{c}
\mathbf{P} \\
1
\end{array}\right]=\left[\begin{array}{c}
\mathbf{Q P}+\mathbf{D} \\
1
\end{array}\right]
$$


where $\mathbf{U}_{\mathbf{A}}, \mathbf{V}_{\mathbf{A}}=$ positional vectors with respect to coordinate frame $\mathrm{A}$; and $\mathbf{T}=$ homogeneous transformation matrix.

In an automatic assembly line, where real-time measurement and robots are used for door fitting, the rotational and translational variables can be used to determine the movement of a robot manipulator to achieve the best fit.

2.2.2. Constrained optimization. The best door-fitting problem is similar to that of part mating, as proposed by Bona and Menga (1984) and Gottschlich and Kak (1989). The problem is now to find the optimal orientation of the door in the three-dimensional space which gives the least variation to the designed nominal gap. The problem can be formulated as a general constrained optimization problem with objective function $J$, subject to equality and inequality constraints.

$$
\begin{aligned}
& \min _{Q} J\left(X_{i}\right) \\
& J\left(X_{i}\right)=\sum_{j} W_{j}\left(\left\|\left(B_{j}-\mathbf{T}\left(X_{i}\right) * A_{j}\right)\right\|-G F_{j}\right)^{2}
\end{aligned}
$$

with equality and inequality constraints

$$
\begin{aligned}
E\left(X_{i}\right) & =0 \\
G\left(X_{i}\right) & \leq 0
\end{aligned}
$$

where $J\left(X_{i}\right)=$ objective function to be minimized; $X_{i}=$ homogeneous transformation variables; $W_{j}=$ weights of importance for each fitting point; $B_{j}=j$ th measurement point on the body; $A_{j}=j$ th measurement point on the door; $G F_{j}=j$ th nominal gap or flush; $\mathbf{T}\left(X_{i}\right)$ $=$ homogeneous transformation matrix $(4 \times 4) ; E=$ equality constraints; $G=$ inequality constraints, for example, tolerances of gaps; and $\|\cdot\|=$ Euclidean norm of a vector.

The objective function in the current study as described is the variation of the distances between the BTW opening dimensions and the corresponding door dimensions from the target gap or flush. In some instances, only one-directional distance is of concern. Under such circumstances, the objective function can be modified by adding a "directional weight" when calculating the weighted distance. Equation (4) shows the variation of point $j$ to its target gap.

$$
J_{j}=\left(\sqrt{\delta_{1 j}\left(x_{B}-x_{A}\right)^{2}+\delta_{2 j}\left(y_{B}-y_{A}\right)^{2}+\delta_{3 j}\left(z_{B}-z_{A}\right)^{2}}-G F_{j}\right)^{2}
$$

where $\delta_{i j}=$ directional weights; $\left(x_{B}, y_{B}, z_{B}\right)=j$ th body side opening point or target point; $\left(x_{A}, y_{A}, z_{A}\right)=j$ th door point; and $G F_{j}=j$ th nominal gap.

Equation (3) then becomes a scalar function as in equation (5).

$$
J\left(X_{i}\right)=\Sigma W_{j} J_{j}
$$


The transformation variables in the current study are the six degrees of freedom kinematic variables of the homogeneous transformation (Asada and By, 1985). In the determination of the homogeneous transformation matrix, the roll, pitch, and yaw angles and the three translational variables are determined with respect to the body coordinate on the door by translating the global origin to a defined body coordinate origin. The body coordinate origin is determined based on the nominals of the fixturing points $\left(X_{\text {net }}, Y_{\text {net }}, Z_{\text {net }}\right)$ as follows:

$$
\left(X_{b 0}, Y_{b 0}, Z_{b 0}\right)=\frac{1}{n}\left[\sum_{i=1}^{n} X_{\text {net } i}, \sum_{i=1}^{n} Y_{\text {net } i}, \sum_{i=1}^{n} Z_{\text {net } i}\right]
$$

The locating points are so constrained such that the adjustment is within the range of feature modeling. For the rest of the checking points, the distances between the door and BIW opening can be specified by the tolerances as inequality constraints. Finally, for locators which may not be adjusted, they will constitute the equality constraints in the problem.

The features of the fitting areas can be defined as either points, lines, or planes, which will be presented in section 3.2 .

\subsection{Simulation and analysis}

The effectiveness of optimal door fitting, measured by the three quality indexes, were evaluated by fitting a rear door to the BIW opening under different conditions of nominal gap and process variation.

The variations in the BIW opening and in the door are assumed to be on the same order of magnitude, and both contribute to the variations of the door gaps. The variations are simulated by superimposing Gaussian white noises to the nominals in $x$-, $y$-, $z$-dimensions with desired mean values for both door and BIW points. The magnitude of variation relative to the nominal gap is measured by the signal-noise ratio $(S / N)$, which is the mean deviation divided by the standard deviation $(\sigma)$ of superimposed noise, that is:

$$
\frac{S}{N}=\frac{\text { magnitude of mean deviation }}{\text { standard deviation of noise }} .
$$

The gap width deviation and gap parallelism for each door are calculated for both with and without optimal fit situations. The average of the GWD and GP for 30 doors is used to evaluate the improvement with optimal fitting on the GWD and $G P$, and the standard deviation of GWD (for 30 doors) is used to evaluate the car-to-car variation.

Without loss of generality, the directional weights are all set to be one, and all the points are treated as equally important.

2.3.1. Within-car variation. The first and second door-fitting dimensional quality requirements, GWD and GP, are studied assuming different nominal gap designs and different process capabilities.

Figure 2 shows the $G W D$ and $G P$ of individual doors before and after optimal fit for 3-mm nominal gaps. It is clear from the figure that the larger the noise (the smaller the $S / N$ ratio), the less the improvement after door fit, especially for gap parallelism. 

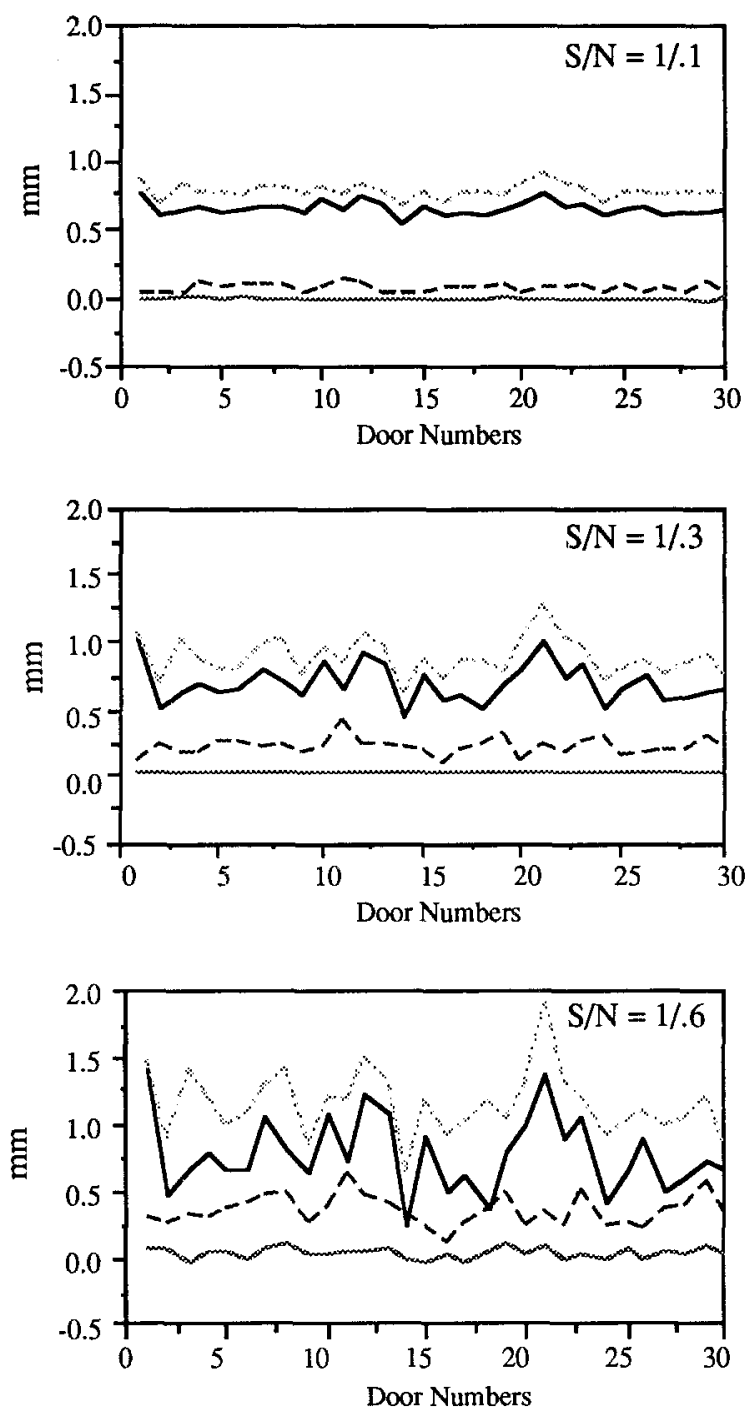

GWD without optimal fit

GWD with optimal fit

........... GP without optimal fit

-- $\quad$ GP with optimal fit

Figure 2. GWD and GP for individual doors with nominal gap $3 \mathrm{~mm}$.

Figures 3 and 4 show the results of averaged $G W D$ and $G P$ before and after optimal fit with respect to different levels of variation. Zero mean deviation is assumed in figure 3 , while a $1-\mathrm{mm}$ mean deviation is assumed for figure 4 . It can be seen from both figures 3 and 4 that when the gap is reduced the variation tends to be more visible. Thus, the 

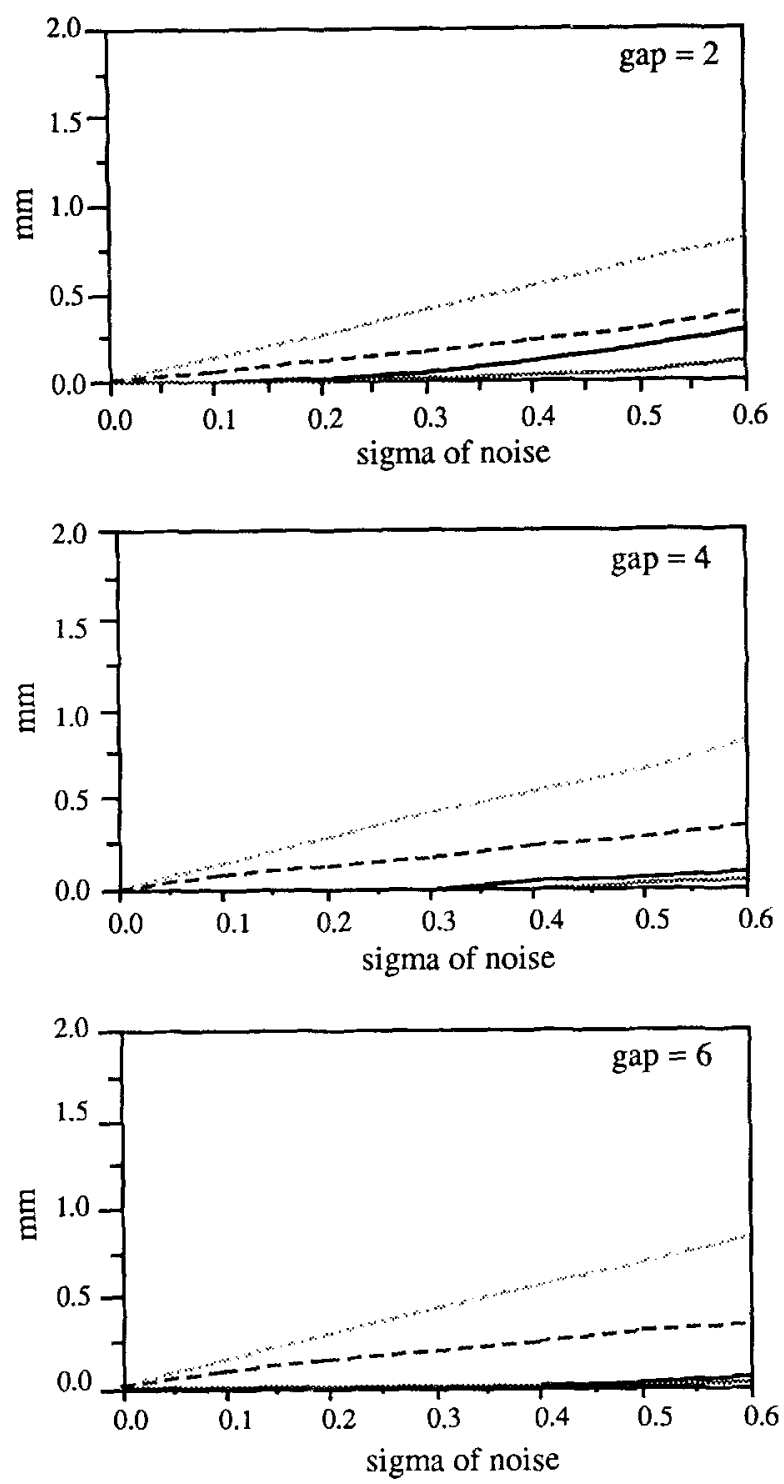

GWD without optimal fit

GWD with optimal fit

GP without optimal fit

- - -. GP with optimal fit

Figure 3. Averaged GWD and $G P$ for zero mean deviation with different nominal gaps.

smaller the gap, the more prominent the variation will be, and consequently higher quality parts with less variations are required for good door fit. The quality after fitting is also found to be affected more by the variation ( $\sigma$ of noise) than by the mean deviation, especially 

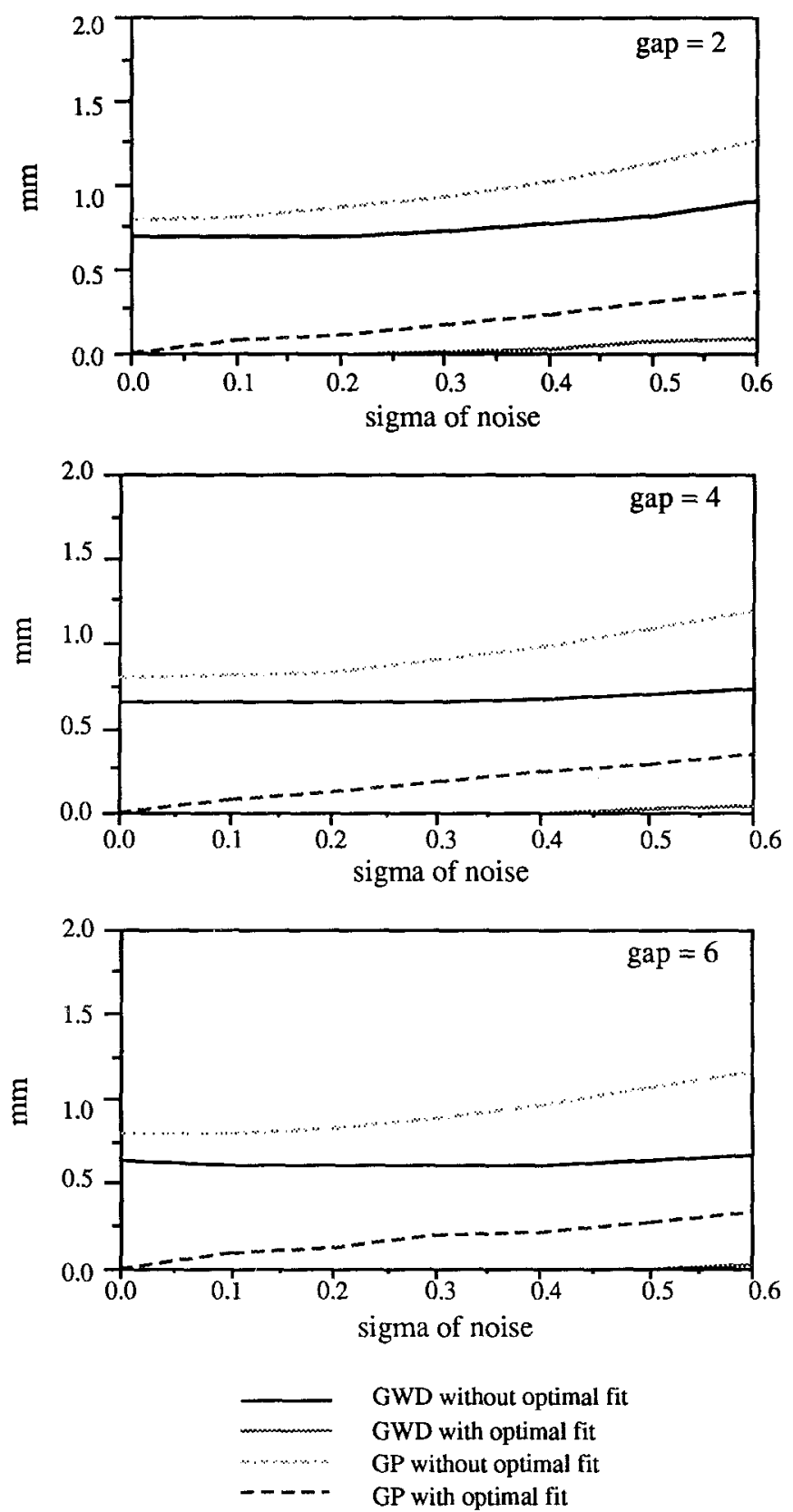

Figure 4. Averaged GWD and GP for 1-mm mean deviation in (-x)-direction.

for the rigid body door movement. Thus, the optimal fitting will be more effective in correcting the process mean deviations than random variations, especially when a smaller nominal gap is designed. 
The necessity of variation reduction can be demonstrated using figure 3 . From the viewpoint of process improvement, the nominal gap is fixed for a given design. Assume the $G W D$ requirement is designated to be $(G W D \leq 0.1 \mathrm{~mm})$, and the gap parallelism is designated to be $(G P \leq 0.2 \mathrm{~mm}$ ) while the nominal door gap is $4 \mathrm{~mm}$. As shown by figure 3 , in order to achieve the quality requirement, the variation ( $\sigma$ of the noise) needs to be controlled under $0.3 \mathrm{~mm}$.

Figure 5 shows the $G W D$ and $G P$ before and after optimal fit of zero mean deviation for different nominal gap designs. From the viewpoint of design, one needs to design the achievable nominal gap based on known process capability. Figure 5 can be used to identify the relationship between variation and nominal gap design. For example, assuming the noise level is $0.3 \mathrm{~mm}$ ( 1 standard deviation) and the quality requirements to be the same as before, from figure 5, the nominal gap must be at least $4 \mathrm{~mm}$ so that the quality level can be achieved after optimal fit. It can also be found that a 6-mm nominal gap can only achieve $0.3 \mathrm{~mm}$ gap parallelism when the process capability ( $\sigma$ of noise) is $0.5 \mathrm{~mm}$.

2.3.2. Batch-to-batch fit vs. $100 \%$ door fitting. The previous discussions are based on $100 \%$ door fitting. When only batch-to-batch fit is implemented, as in the case of manual operation, the mean value of a batch of doors will be used for optimal fitting. Under such
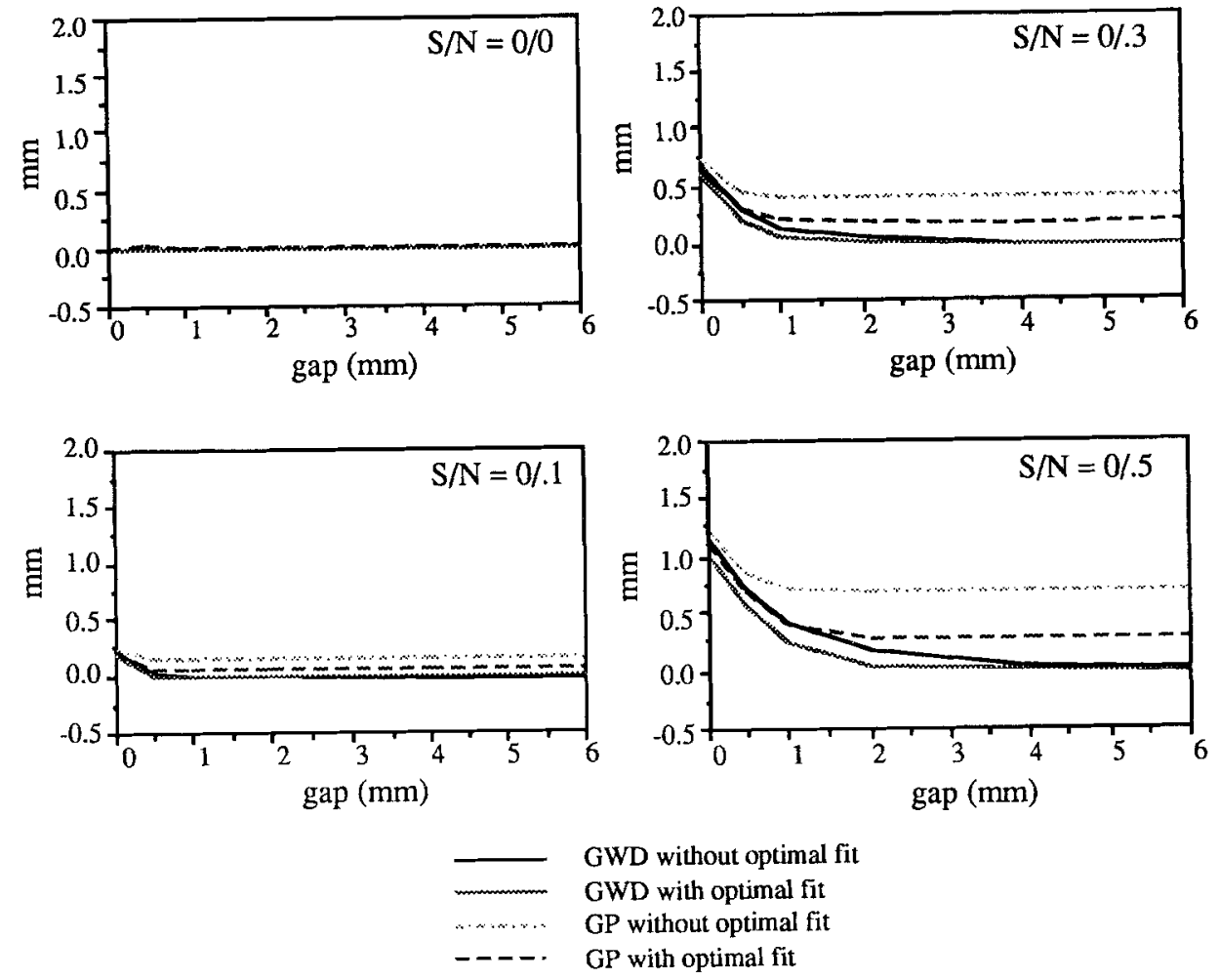

Figure 5. Averaged $G W D$ and GP for different noise levels. 
circumstances, only the mean deviations can be corrected by optimal door fitting while the door-to-door variation still remains. The $G W D$ and $G P$ curves before optimal door fit in figure 3 for zero mean deviation provide the best gap quality possible for batch-to-batch fit. Therefore, the difference between the curves before fit and the curves after fit in figure 3 shows the difference between batch-to-batch fit and 100\% optimal door fit.

2.3.3. Car-to-car variation. The third door-fitting criterion is the consistency of door gap widths from car to car, which is measured by the standard deviation of GWD. The $\sigma$ of $G W D$ vs. different nominal gaps for several $S / N$ ratios are shown in figure 6. Taking the
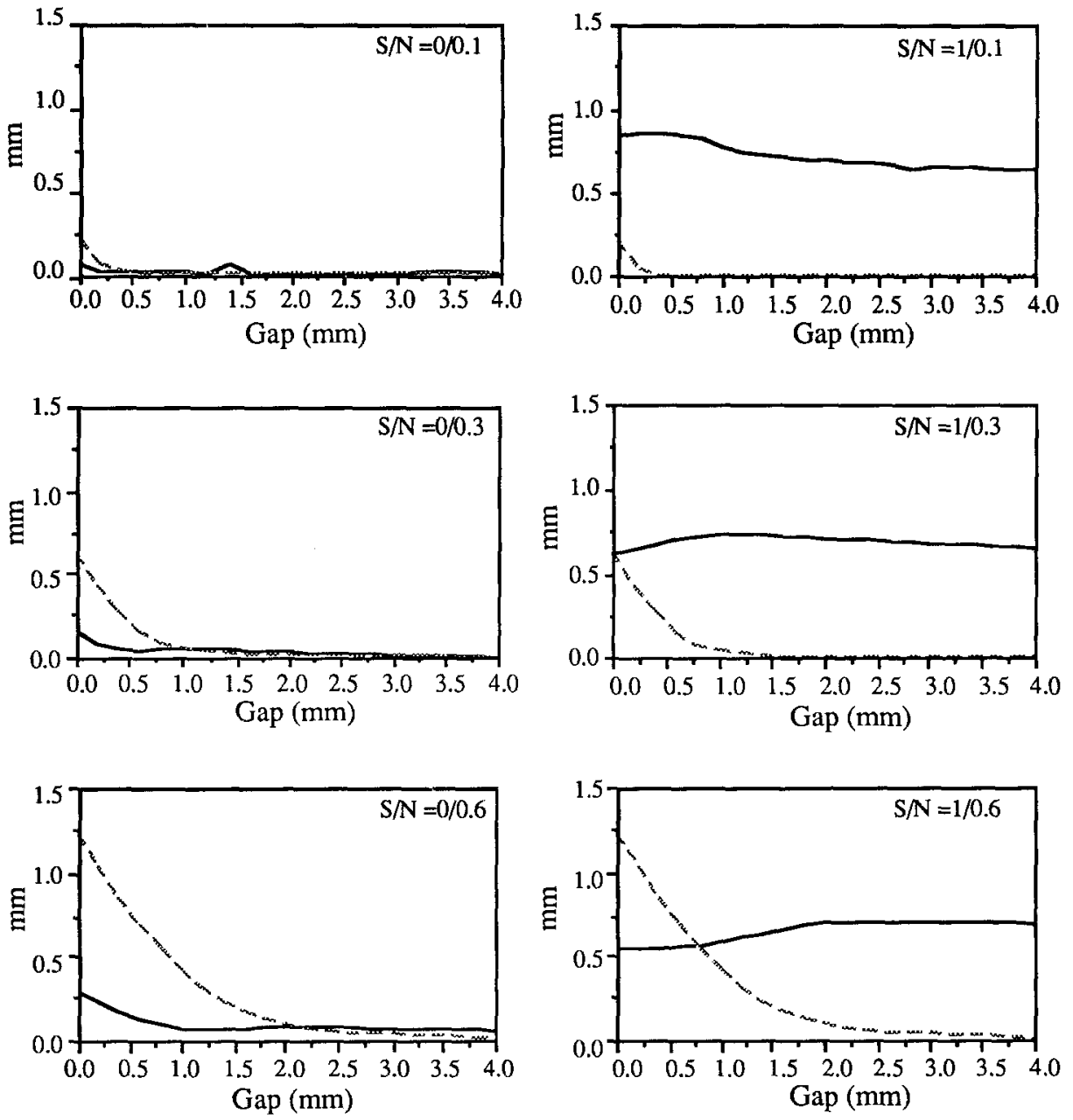

- Before Fit

Figure 6. $\sigma$ of GWD for $0-\mathrm{mm}$ and 1-mm mean deviation. 
zero mean deviation case as an example $(S / N=0 / \sigma)$, the $G W D$ s tend to be more severe for smaller nominal gaps. Therefore, when the gap is small, the GWDs between cars are "consistently bad" before optimal fit. After optimal fit, although the GWDs are improved as in figures 3-5, the quality tends to be "inconsistently good." This explains the larger $\sigma$ of $G W D$ after optimal fit in the small nominal gap region.

When there is a mean deviation, for example, $1 \mathrm{~mm}$ to the fore direction, it is equivalent to a widening of the gap. A wider gap resulted in better variation tolerating capability. Thus, it is seen in figure 6 that the curves of before fit and after fit intersect when the $\sigma$ of noise is larger than $0.3 \mathrm{~mm}$, and the point of intersection changed from 2-mm nominal gap without mean deviation to $0.8-\mathrm{mm}$ nominal gap with $1-\mathrm{mm}$ deviation.

\section{A systematic fixture adjustment scheme}

The realization of the optimal door fit requires adequate adjustments of the door hanging fixture. A door hanging fixture with its locators, or nets for two-directional positioning is shown in figure 7. Conventionally, the adjustment of fixture for door fitting is based on trial and error. Also, the local features of the door in the contacting areas are not considered in fixture adjustment. For example, when a door is found to be "high," the H/L locator in $z$-direction is adjusted as shown in figure 8(a). The local featrues of the other locators, such as the $x$-directional locator in figure 8(a), are considered to be irrelevant to the part movement. This assumption of irrelevance will be inadequate if the local feature of $x$ directional locator is not perpendicular to the direction of part movement as shown in figure 8(b). Adjustment of the $z$-directional locator alone will either cause incorrect door movement or indent the contact surface of the door. Thus, the local features of the surfaces around the locating point need to be considered in the determination of fixture adjustment.

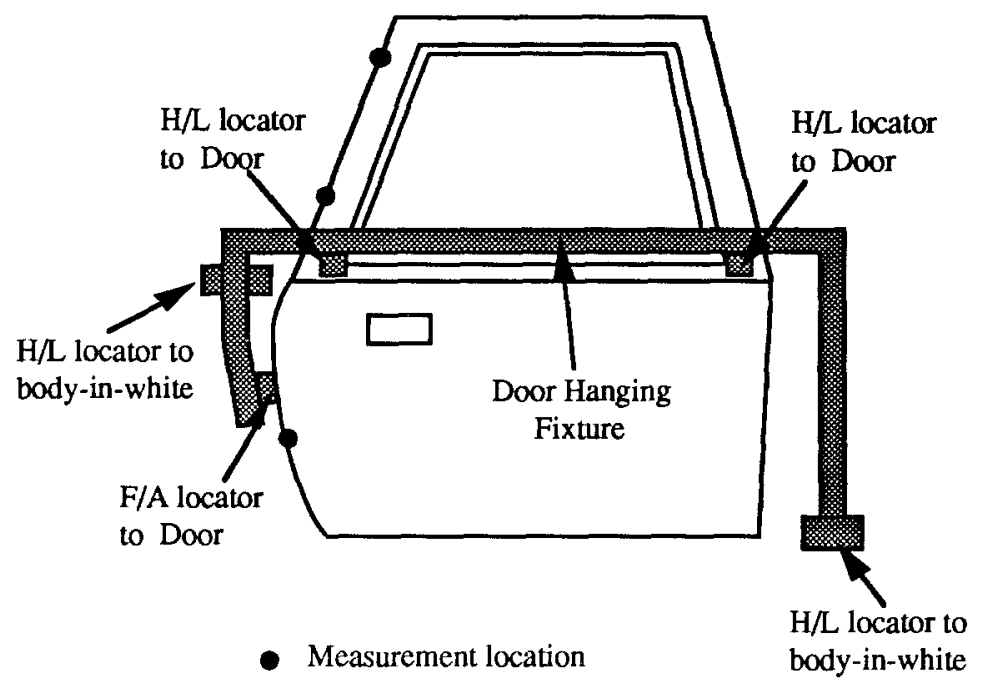

Figure 7. Door hanging fixture and F/A measurement location on a door. 


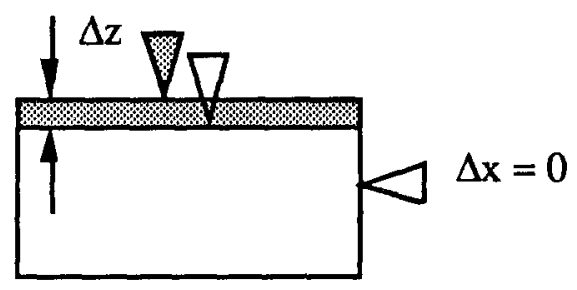

(a)

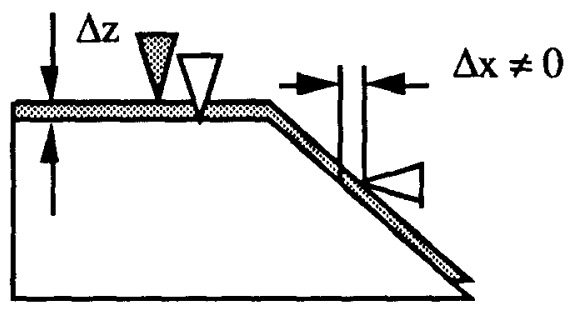

(b)

Figure 8. Error in locating when local feature is not considered.

Take a point locator as an example. The door surface in contact with the locator needs to remain in contact after the locator being adjusted, as shown in figure 9. After the adjustment, the contact point on the surface will move to a new location in the neighborhood of the original contact point. The local surface feature around the original contact point needs to be modeled to determine the location of the new contact point and the amount of adjustment.

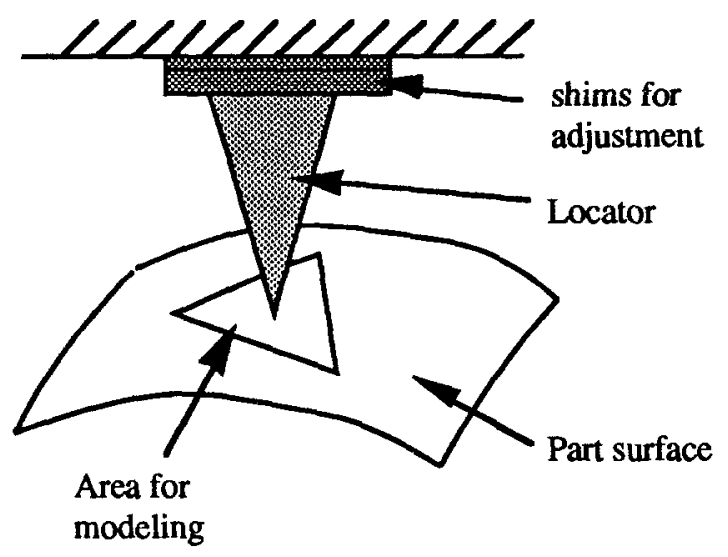

Figure 9. A locator in contact with part surface. 
A systematic scheme to determine the fixture adjustment considering the netting features is presented as follows.

\subsection{Geometric modeling of local features}

3.1.1. Parametric modeling of geometry. A spatial surface can generally be represented using a parametric algebraic form (Mortenson 1985; Hoffmann 1989) as in equation (7). Here $a_{i j}, b_{i j}$, and $c_{i j}$ are the coefficients to describe the surface. Parameters $u$ and $w$, when assigned fixed values, will represent a specific point on the modeled surface. For the case when $m=n=3$, equation (7) becomes the bicubic representation of a surface patch. When $n=0$ in equation (7), the spatial surface equation simplifies to a spatial curve, which is now a function of parameter $u$ only. Again $m$ needs to be determined depending on the complexity of the curve.

$$
P(u, w)=\left\{\begin{array}{l}
X(u, w) \\
Y(u, w) \\
Z(u, w)
\end{array}\right\}=\left\{\sum_{i=0}^{m} \sum_{j=0}^{n} P_{i j} u_{i} w_{j}\right\}=\left\{\begin{array}{c}
\sum_{i=1}^{m} \sum_{j=0}^{n} a_{i j} u^{i} w^{j} \\
\sum_{i=0}^{m} \sum_{j=0}^{n} b_{i j} u^{i} w^{j} \\
\sum_{i=0}^{m} \sum_{j=0}^{n} c_{i j} u^{i} w^{j}
\end{array}\right\}
$$

3.1.2. Geometry parametric fitting using CMM data. To obtain the local feature around a contact point, data from a coordinate measuring machine (CMM) were used for feature model fitting. Using the general bicubic surface as an example, the objective is to obtain the parameters $a_{i j}, b_{i j}$, and $c_{i j}$ by measuring some points $\left(X_{i}, Y_{i}, Z_{i}\right)$ on the surface to be modeled. These parameters can then be estimated by minimizing the distances between the measured dimensions $\left(X_{i}, Y_{i}, Z_{i}\right)$ and their algebraic parameteric form, as in equation (8).

$$
\min _{a_{i j}, b_{i j}, c_{i j}} J\left(a_{i j}, b_{i j}, c_{i j}\right)=\sum_{k=1}^{n p}\left\|\mathbf{P}_{k}-\sum_{i=0}^{m} \sum_{j=0}^{n} \mathbf{P}_{i j} u_{k}^{i} w_{k}^{i}\right\|^{2},
$$

where $k$ is the $k$ th measured point and $n p$ is the total number of points used for fitting. The number of points needed for fitting, $n p$, is different depending on the assumptions about the local feature. For the surface represented by equation $(7), 3 \cdot(m+1) \cdot(n+1)$ parameters of $a_{i j}$ and $b_{i j}$ need to be determined. Each measured point provides three equations. Thus the number of points required to fit a surface of equation (7), $n p$, can be determined by:

$$
\begin{gathered}
3 n p \geq 3((m+1) \cdot(n+1)), \text { or } \\
n p \geq(m+1) \cdot(n+1) .
\end{gathered}
$$


Similarly, the number of points needed to fit a curve is:

$$
n p \geq(m+1) \text {. }
$$

When the number of points used is more than the number of points needed, the minimization problem is in the sense of a least squares fit.

Since the adjustment of a door on the body side opening is usually small, the local feature can generally be modeled as a planar surface unless there is a large curved surface for a specific body type. A planar surface, which is assumed for the body type under study, can always be represented by a point and two vectors on the surface. The general bicubic representation of the surface described by equation (7) then simplifies to equation (11). In equation $(11), P(0,0)$ is the original locating datum. $P(1,0)$ and $P(0,1)$ are the two nonparallel vectors on the surface which are determined by measuring three points $3 \mathrm{~mm}$ away from the locating datum as shown in figure 10. For the edge in F/A locating, nine points $3 \mathrm{~mm}$ apart in $z$-direction are measured for cubic curve modeling, as shown in figure 11 .

$$
\mathbf{P}(u, w)=\left\{\begin{array}{l}
X(u, w) \\
Y(u, w) \\
Z(u, w)
\end{array}\right\}=\mathbf{P}(0,0)+\mathbf{P}(1,0) \cdot u+\mathbf{P}(0,1) \cdot w
$$

\subsection{Determination of fixture adjustment}

In this section, three commonly used locator/part combinations for door hanging are analyzed; they are: (1) a locator point in contact with a part surface; (2) a locator surface with a part surface; and (3) a locator surface with a part edge (spatial curve).

3.2.1. A locator point with a part surface. The point in contact with a part surface in part fixturing was shown in figure 9. After the local feature is properly modeled, the corresponding adjustment for a point locator can be determined by equation (12).
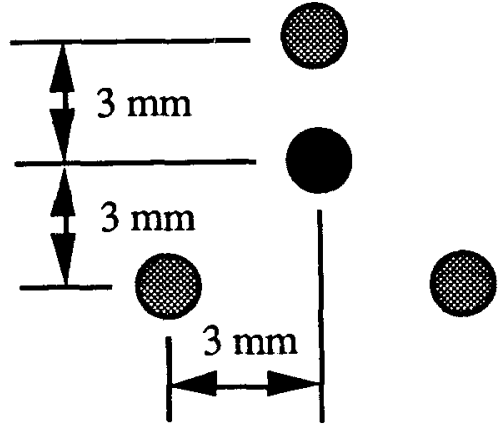

Figure 10. Datum measurement for surface feature modeling. 


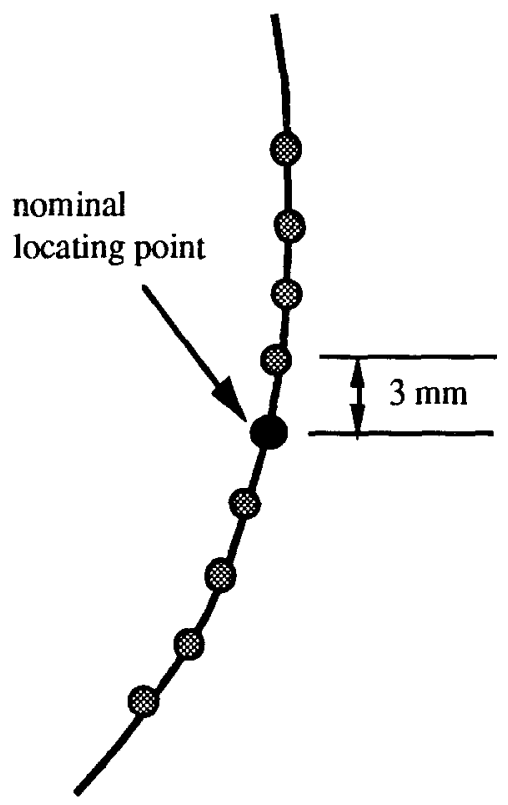

Figure 11. Datum measurement for edge modeling.

$$
\mathbf{T} \cdot\left\{\begin{array}{c}
X(u, w) \\
Y(u, w) \\
Z(u, w) \\
1
\end{array}\right\}=\left\{\begin{array}{c}
X(\text { net })+K \cdot a \\
Y(\text { net })+K \cdot b \\
Z(\text { net })+K \cdot c \\
1
\end{array}\right\}
$$

where $\mathbf{T}=$ homogeneous transformation matrix; $(a, b, c)^{\prime}=$ unit vector in the direction of adjustment; $K=$ amount of adjustment; and $u, w, K=$ variables.

3.2.2. A surface locator with A part surface. A block with a locating surface rather than a point is frequently used for part fixturing as shown in figure 12 . When the door is oriented for optimal door fit, the amount of adjustment is determined by minimizing the distance between the oriented door and the NC block surface in the adjustment direction, as shown in figure 13.

When the two contact surfaces are relatively convex to each other as shown in figure 14 , a unique solution is readily available by minimizing the distance between the transformed door surface and the locator block surface in the direction of adjustment subject to the constraint $G((x(u, w), y(u, w), z(u, w)) \leq$ (or $\geq 0)$. Here $G(x, y, z)$ is the explicit form of the locator block surface. The objective function $J$ to be minimized is equation (13).

$$
J=\left\|\mathbf{T} *\left\{\begin{array}{c}
X_{\text {part }}(u, w) \\
Y_{\text {part }}(u, w) \\
Z_{\text {part }}(u, w) \\
1
\end{array}\right\}-\mathbf{T}_{\text {net }} *\left\{\begin{array}{c}
X_{\text {net }}(s, t) \\
Y_{\text {net }}(s, t) \\
Z_{\text {net }}(s, t) \\
1
\end{array}\right\}\right\|^{2},
$$




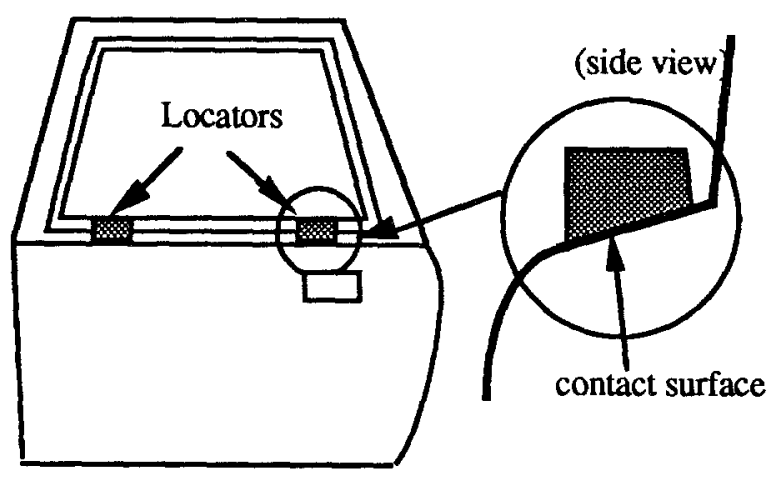

Figure 12. Locating block results in a contact surface instead of point contact.
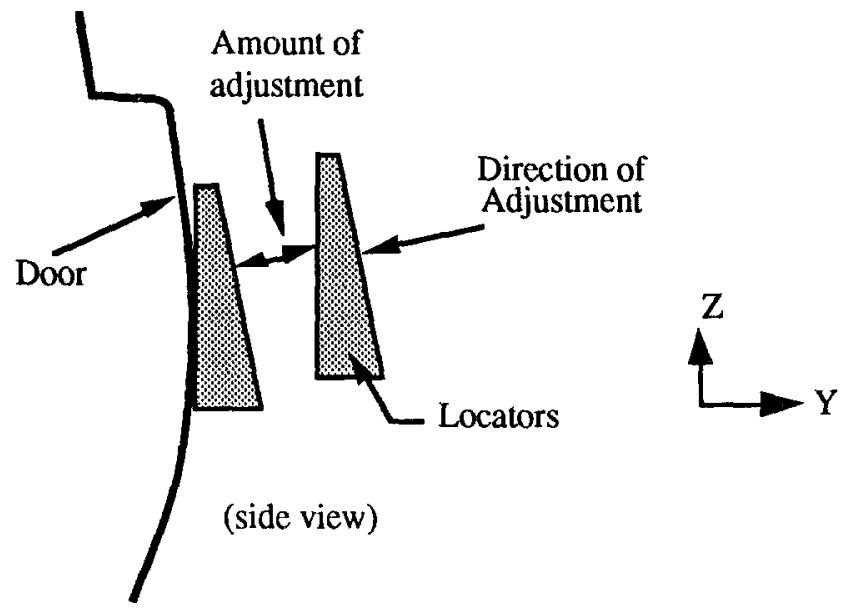

Figure 13. Finding amount of adjustment in the adjustment direction.

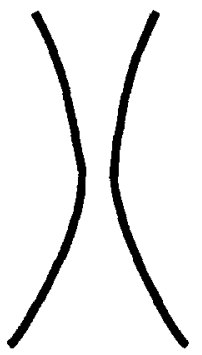

(a)

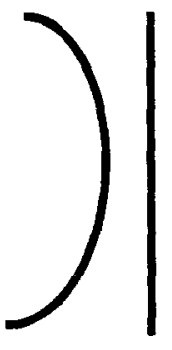

(b)

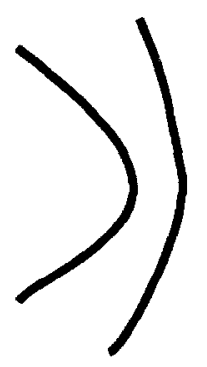

(c)

Figure 14. Relatively convex surfaces (side view). 
where $\mathbf{T}=$ known homogeneous transformation matrix for optimal door fit

$$
\mathbf{T}_{\text {net }}=\left[\begin{array}{cccc}
1 & 0 & 0 & a \cdot K \\
0 & 1 & 0 & b \cdot K \\
0 & 0 & 1 & c \cdot K \\
1 & 1 & 1 & 1
\end{array}\right]
$$

which is the transformation matrix for locator adjustment in the specified direction; $(a, b, c)^{\prime}$ $=$ unit vector defining the direction of adjustment; $K=$ amount of adjustment; $X(\#, \cdot)=$ parametric representation of $X$ in $\#$ and $\cdot$, same for $Y$ and $Z$; and $\|\cdot\|=$ Euclidean norm.

The procedure for finding the locator adjustment can be summarized as follows:

1. Model the contact surface in parametric form,

2. Obtain the optimal orientation of the door, $\mathbf{T}$,

3. Transform the parametric representation of the door surface, and

4. Find the amount of adjustment (figure 13) by minimizing the distance between the transformed door surface and the locator block in the direction of adjustment.

For most applications in door fixturing, a rectangular planar surface is designed for the part positioning. The problem is then simplified to solving four point-surface contact problems as was described in section 3.2.1 for the four boundary corners. The corner which gives the minimal distance provides the solution for the amount of adjustment.

3.2.3. A locator surface with A part edge. When the part to be fixtured has a feature of a curved edge (F/A locating area in the door), the point locator will be inadequate because it will no longer be possible for the locator to be in contact with the edge after the part is oriented. A locator block with a planar surface is usually implemented in such circumstances. A graphical description of the edge fixturing is shown in figure 15 .

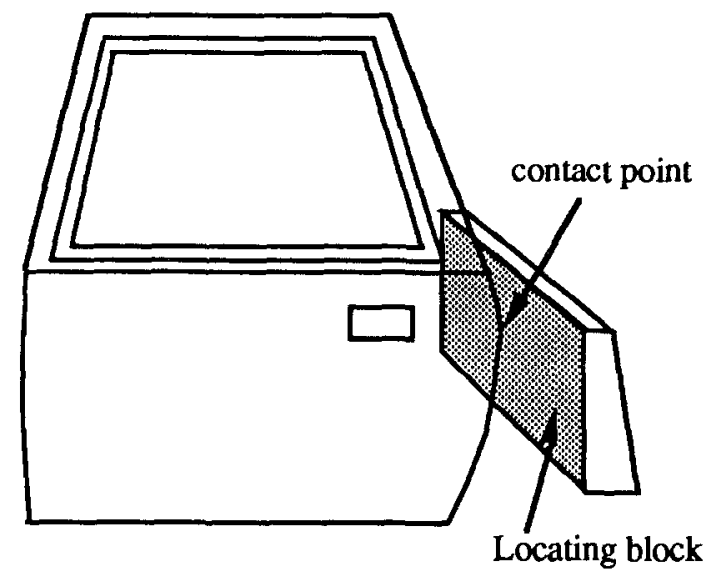

Figure 15. Locating block in contact with an edge of a door. 
The procedure to find the amount of adjustment for the net to achieve the optimal door fit is as follows:

1. Model the curved edge of part in parametric form,

2. Model the surface of locating block in parametric form,

3. Model the boundary of the locating block,

4. Transform the parametric representation of the edge curve,

5. Find the amount of adjustment (similar to figure 13) by minimizing the distance $J$ between the transformed curve and the locating block surface in the direction of adjustment (equation (14)) subject to the constraint of $G((x(u), y(u), z(u)) \geq 0$ for the right door, if the edge and the locating surface is relatively convex (here, $G(x, y, z)$ is the explicit form of the locating surface), and

6. Find the amount of adjustment by minimizing $J$ in equation (15) if the edge and the locating surface is not relatively convex.

$$
\begin{aligned}
& J=\left\|\mathbf{T} *\left\{\begin{array}{c}
X_{\text {part }}(u) \\
Y_{\text {part }}(u) \\
Z_{\text {part }}(u) \\
1
\end{array}\right\}-\mathbf{T}_{\text {net }} *\left\{\begin{array}{c}
\mathrm{X}_{\text {net }}(s, t) \\
Y_{\text {net }}(s, t) \\
Z_{\text {net }}(s, t) \\
1
\end{array}\right\}\right\|^{2}, \\
& J=\left\|\mathbf{T} *\left\{\begin{array}{c}
X_{\text {part }}(u) \\
Y_{\text {part }}(u) \\
Z_{\text {part }}(u) \\
1
\end{array}\right\}-\mathbf{T}_{\text {net }} *\left\{\begin{array}{c}
\mathrm{X}_{\text {net }}(s) \\
Y_{\text {net }}(s) \\
Z_{\text {net }}(s) \\
1
\end{array}\right\}\right\|^{2},
\end{aligned}
$$

where $(X(u), Y(u), Z(u))=$ parametric representation of the door edge.

\subsection{Door-fixture-body adjusting mechanism}

To hang a door on the body side opening, the door is first positioned on the fixture using locators between the door and fixture. The door-fixture is then positioned on the body side opening using locators between the fixture and BIW. Thus there are two adjusting mechanisms: adjustment between door and fixture, and adjustment between fixture and body. The schematic representation of the two locating mechanisms are shown in figure 16(a). When the amount of door translations and rotations are determined, either one of the adjusting mechanisms can be used to achieve the desired door orientation. The adjustment of the two mechanisms are discussed as follows.

3.3.1. Adjustment between door and hanging fixture. To achieve the desired door orientation, the door is first computationally oriented in the space. The amount of adjustment for the locators on the fixture are then determined by extending/retracting the locators until they touch the door surfaces again. Figure 16(b) shows the adjustment of locators between door and fixture while the fixture-body locators remain unchanged. Derivations in previous sections for fixture adjustment are thus for the door-fixture mechanism. 


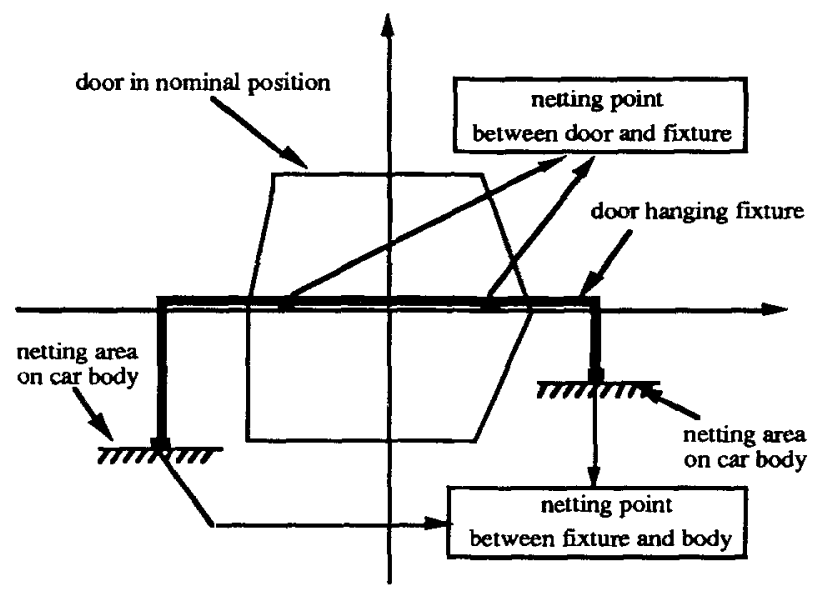

Figure 16(a). Locators or nets for door-fixture and fixture-body.

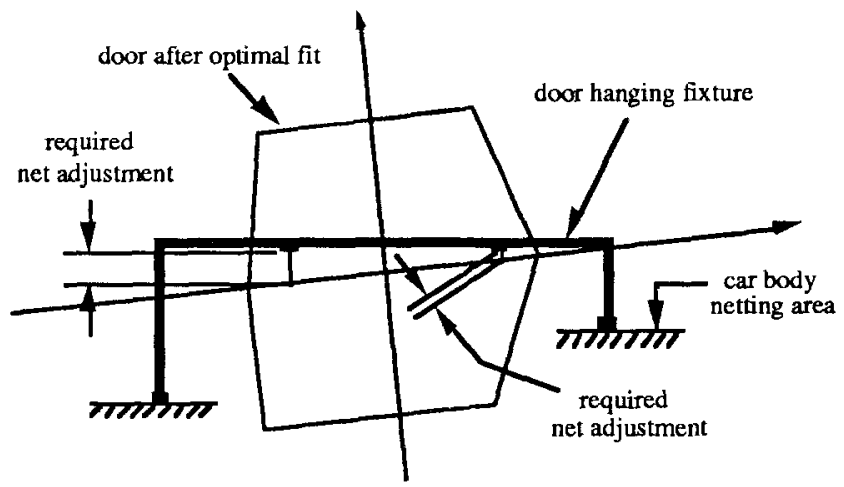

Figure 16(b). Adjustment of nets between door and fixture.

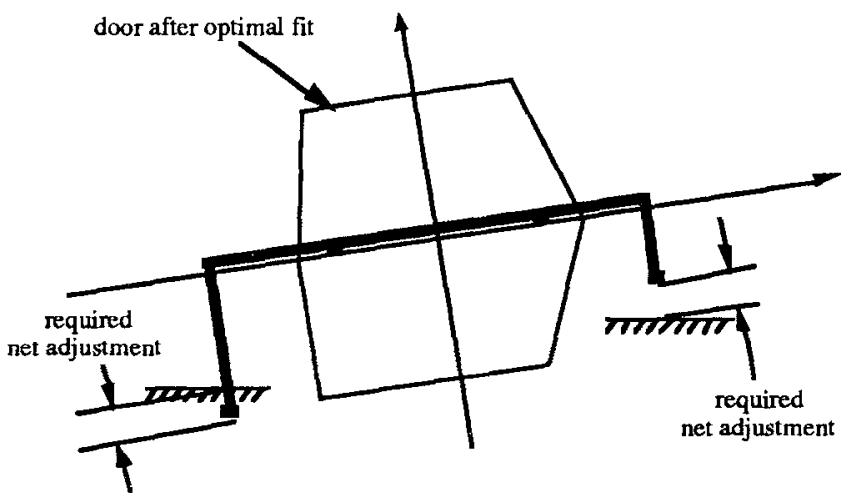

Figure 16(c). Adjustment of nets between fixture and body. 
3.3.2. Adjustment between hanging fixture and BIW. When the locators between the fixture and BIW are used for door-fitting adjustment, the fixture frame is now being oriented in the space together with the door as in figure 16(c). Once this is done, the locators are computationally extended to touch the body locating surface and the amount of adjustment determined.

Since the orientation of the door relative to the car body can be treated as the inverse orientation of the body to the door, the problem can be solved in a similar way as before except that the determination of the locator adjustment is done by orienting the body rather than the door. The procedure is as follows:

1. Model the surface feature in the contact area of the car body,

2. Determine the desired door orientation by homogeneous transformation,

3. Find the inverse transformation of step 2, and

4. Multiply the body surface feature with the inverse transformation and solve for the amount of adjustment as equation (16).

$$
\mathbf{T}^{-1} \cdot\left\{\begin{array}{c}
X(u, w) \\
Y(u, w) \\
Z(u, w) \\
1
\end{array}\right\}=\left\{\begin{array}{c}
X(\text { net })+K \cdot a \\
Y(\text { net })+K \cdot b \\
Z(\text { net })+K \cdot c \\
1
\end{array}\right\}_{\text {body }}=
$$

where $\mathbf{T}^{-1}=$ inverse transformation of $\mathbf{T} ; K=$ amount of adjustment; and $(a, b, c)^{\prime}=$ unit vector defining the direction of adjustment.

\subsection{Experimental verification}

The objective of fixture adjustment is to fit the door to the side opening to obtain the best gap and flush dimensional quality possible. The presented door-fitting approach includes (1) the determination of the desired door orientation; and (2) the determination of fixture adjustment to achieve the door orientation. The determination of door orientation is a straightforward optimization based on door and body measurements, which demands no verification if the measurements are reliable. The determination of fixture adjustment, however, involves local feature modeling in the netting area, and needs to be verified through experiment.

To verify the local feature modeling, intuitively one wants to measure the dimensions before fit, find the desirable fixture adjustment, and measure the dimensions after fit to confirm the result of fixture adjustment. Since only the fixture adjustment needs to be verified, an inverse verification is incorporated here to simplify the experimental procedure. The inverse verification is to conduct a predetermined fixture adjustment, and compare the computed dimensions with the measured dimensions to confirm the local feature modeling. An experiment was conducted on the production floor to verify the modeling of the rear edge of a door for F/A fixturing.

A right-hand rear door, a BIW, and a door hanging fixture for production are used for the experiment in the body assembly plant. The door hanging fixture and the checkpoints 
on the door are shown in figure 7. The resolution of the hanging fixture was usually $0.25 \mathrm{~mm}$ (the thickness of the smallest shim used for production), even though $0.1-\mathrm{mm}$ resolution was possible. The door-fitting experiment, the computational detail, and the comparison of the results are discussed in the following sections.

3.4.1. Door-fitting experiments. Two sets of evaluations were conducted with the door hung and measured on the body side opening by the hanging fixture. The general experimental procedure is:

1. Put the BIW on the layout plate (similar to a "manual CMM"),

2. Conduct shimming on the $\mathrm{H} / \mathrm{L}$ nets of the door hanging fixture,

3. Hang the door on the body side opening using the hanging fixture and tighten the bolts at hinge to the side opening, and

4. Measure the door dimensions at the checking points.

The door was first hung on the side opening and measured without shimming. The dimensions measured without shimming were then used as the datum for computation. A 2-mm shim was then added to both $\mathrm{H} / \mathrm{L}$ nets in the fixture, causing the door to move down $(-2 \mathrm{~mm})$, and then the door was bolted on the body and again its positions measured for comparison.

3.4.2. Computation. The F/A door edge and its planar locator surface were modeled first. The modeled surface and edge models are:

1. Locator surface

$$
\left\{\begin{array}{c}
X(s, t) \\
Y(s, t) \\
Z(s, t)
\end{array}\right\}=\left[\begin{array}{rrr}
4296.37 & 0 & 5.71 \\
961.90 & 1 & 0.27 \\
864.75 & 0 & 11.9
\end{array}\right]\left\{\begin{array}{l}
1 \\
s \\
t
\end{array}\right\}
$$

2. Cubic curve of rear door edge

$$
\left\{\begin{array}{l}
X(u) \\
Y(u) \\
Z(u)
\end{array}\right\}=\left[\begin{array}{rccc}
4206.3703 & 5.7103 & -0.1563 & -0.0436 \\
961.9024 & 0.2726 & -0.0955 & -0.0241 \\
864.7421 & 11.9972 & 0.000029 & -0.0324
\end{array}\right]\left\{\begin{array}{c}
1 \\
u \\
u^{2} \\
u^{3}
\end{array}\right\} .
$$

The H/L locators, being far apart from each other, were assumed to be point contacts. The amount of adjustments in the $\mathrm{H} / \mathrm{L}$ locators were substituted into equation (12) for $K \mathrm{~s}$ solved simultaneously with equation (14) for the homogeneous transformation matrix. The F/A checking points before shimming were then multiplied to the homogeneous transformation matrix and also tabulated as the calculated dimensions in table 1.

Shimming by $2 \mathrm{~mm}$ for both $\mathrm{H} / \mathrm{L}$ locators is equivalent to lowering the door. Due to the feature in the F/A edge, as can be seen in figure 7, the door will be pushed forward in addition to a simple $\mathrm{H} / \mathrm{L}$ movement. From table 1, the upper and lower measured points were found to be very consistent with the calculated ones after shimming. The central point, 
Table 1. Measured and calculated F/A dimensions due to shimming.

\begin{tabular}{|c|c|c|c|c|c|c|}
\hline & \multirow{2}{*}{$\begin{array}{c}\text { Net } 1 \\
(0 \mathrm{~mm},\end{array}$} & \multirow{2}{*}{$\begin{array}{c}\text { Net } 2 \\
0 \mathrm{~mm}) \\
\text { Calculated }\end{array}$} & \multirow{2}{*}{$\begin{array}{c}\text { Net } 1 \\
(-2 \mathrm{~mm}, \\
\text { Measured }\end{array}$} & \multirow{2}{*}{$\begin{array}{c}\text { Net } 2 \\
-2 \mathrm{~mm}) \\
\text { Calculated }\end{array}$} & \multicolumn{2}{|c|}{$\begin{array}{l}\text { Resultant Movement } \\
(\mathrm{mm})\end{array}$} \\
\hline & & & & & Measured & Calculated \\
\hline Upper point & 4099.0 & . & 4907.8 & 4097.766 & 1.2 & 1.234 \\
\hline Middle point & 4225.4 & - & 4223.7 & 4224.279 & 1.7 & 1.121 \\
\hline Lower point & 4250.9 & - & 4249.87 & 4249.811 & 1.1 & 1.089 \\
\hline
\end{tabular}

being in the way of the measuring arm of the layout plate, showed a $0.58-\mathrm{mm}$ difference and can be considered as measuring error. The result thus verified that the modeling of F/A edge netting is adequate.

\section{Conclusions}

The door-fitting problem was formulated as a constrained optimization problem in this paper. Three indexes: (1) door gap width deviation relative to design nominal; (2) door gap parallelism; and (3) car-to-car gap consistency; were defined to evaluate the door gap quality quantitatively. Simulations were conducted to evaluate the optimal fitting on the improvement door gap quality. Based on these simulations, the following observations can be made:

1. The quality of the gap is influenced more by the variation of the door (and the openings) than by the average deviation. Therfore, reducing the variation of the door is important to obtain quality gap.

2. Fitting individual doors to the door openings can improve the quality of fit. Batch-tobatch door fit can only correct for process mean deviations.

3. The size of the designed nominal gap should be process driven. The smaller the nominal gap, the more capable the door manufacturing processes should be.

To achieve the optimal fit, a systematic tooling adjustment determination scheme was then proposed to achieve the desired door orientation for best door fit. The tooling adjustment scheme considered both the geometric features of part and the locators around the netting area for exact fixturing. The geometric modeling of a door edge for F/A fixturing was experimentally verified in a production environment. The results showed good consistency between computed dimensions and measured dimensions.

\section{References}

Asada, H. and By, A.B., "Kinematics of Workpart Fixturing," Proceedings of the 1985 IEEE International Conference on Robotics and Automation, St. Louis, MO, pp. 337-345 (March 1985). IEEE Computer Society Press, Washington, D.C.

Bona, B. and Menga, G., "Best Fitting of Three-Dimensional Bodies," IFAC 9th International World Congress, Budapest, Hungary, pp. 2481-2487 (1984). Published by Pergamon Press, Oxford. 
Gottschlich, S.N. and Kak, A.C. "A Dynamic Approach to High-Precision Parts Mating," IEEE Transactions on Systems, Man, and Cybernetics, Vol. 19, No. 4, pp. 797-810 (July/August 1989).

Hoffmann, C.M. Geometric \& Solid Modeling, An Introduction, Morgan Kaufmann Publishers, Inc., San Mateo, CA (1989).

$\mathrm{Hu}$, S.J., Wu, S.K. and Wu, S.M. "Multivariate Analysis and Variation Reduction Case Studies in Automobile Assembly," Transactions of the North American Manufacturing Research Institute of SME, Vol. 19, pp. 303-308 (1991).

Mortenson, Michael E., Geometric Modeling. John Wiley \& Sons, NY (1985).

Paul, Richard P., Robot Manipulators: Mathematics, Programming, and Control, The MIT Press, Cambridge, MA (1981). 